\title{
ATIVIDADE FARMACOLÓGICA DE ÓLEOS ESSENCIAIS NO SISTEMA RESPIRATÓRIO: UMA REVISÃO SISTEMÁTICA DE ESTUDOS PRÉ-CLÍNICOS
}

Pharmacological activity of essential oils in respiratory system: a systematic review of pre-clinical studies Actividad farmacológica de los aceites esenciales en el sistema respiratorio: una revisión sistemática de estudios preclínicos

Pedro Modesto Nascimento Menezes', Mariana Coelho Brito², Angélica Maria Lucchese ${ }^{3}$, Julianeli Tolentino de Lima ${ }^{1,2}$, Luciano Augusto de Almeida Ribeiro ${ }^{1,2}$, Fabrício Souza Silva ${ }^{1,2^{*}}$

${ }^{1}$ Universidade Federal do Vale do São Francisco

2 Colegiado de Ciências Farmacêuticas, Universidade Federal do Vale do São Francisco

${ }^{3}$ Laboratório de Química de Produtos Naturais e Bioativos

*E-mail: fssilvafarma@gmail.com

\section{Abstract}

Introduction:Essential oils are a mixture of various volatile compounds, which are extracted from aromatic plants and have several pharmacological properties. Among the pharmacological properties described in the literature for these essential oils highlight effects such as insect repellent, anti-inflammatory, antiasthmatic, anxiolytic and anticancer.

Objective: In this review, studies that described the pharmacological effects of essential oils in experimental models involving the investigation of spasmolytic, antiasthmatic, antitussive and/or expectorant activities were analyzed.

Methods: The papers with the keywords "essential oil" and "spasmolytic", "essential oil" and "airway smooth muscle", "essential oil" and "anthiasthmatic", "essential oil" and "antitussive", "essential oil" and "cough", "essential oil" and "mucolytics", "essential oil" and "expectorant" were selected after searching in Pubmed/Medline, Scopus and Biblioteca Virtual de Saúde databases.

Results: Among 868 works identified, which contained keywords in the title and/or abstract, 22 papers were selected and 28 species of plants with studies related to activity of essential oils in the respiratory system.

Conclusion: After analyzing the articles, it was observed that the essential oils are an important source of natural products with pharmacological effect in the respiratory system, justifying the popular use of plant species in the treatment of asthma, cough and to facilitate sputum.

Keywords: Essential oils. Asthma. Cough. Expectoration. Spasmolytic effect.

Resumen

Introducción: Los aceites esenciales son una mezcla de varios compuestos volátiles extraídos de plantas aromáticas y que tienen diversas propiedades farmacológicas. Entre las propiedades farmacológicas descritas en la literatura para esos aceites esenciales, se destaca el efecto repelente, anti-inflamatorios, anti-asmáticos, ansiolíticos y anti-cáncer.

Objetivo: En esta revisión, se analizaron trabajos que relataron los efectos farmacológicos de aceites esenciales en modelos experimentales involucrando la investigación de la actividad espasmolítica, antiasmática, antitusígena y/o expectorante.

Métodos: Los artículos fueron seleccionados después de una búsqueda en las bases de datos Pubmed/Medline, Scopus y Biblioteca Virtual de Saúde con las siguientes palabras clave: essential oil" y "spasmolytic", "essential oil" y "airway smooth muscle", "essential oil" y "anthiasthmatic", "essential oil" y "antitussive", "essential oil" y "cough", "essential oil" y "mucolytics" o "essential oil" y "expectorant". 
Resultado: Entre 868 artículos identificados, que contenían las palabras clave en el título y/o resumen, se seleccionaron 22 trabajos que contenían relatos de 28 especies de plantas con estudios relacionados a la actividad de aceites esenciales en el sistema respiratorio.

Conclusión: Después del análisis de los artículos, se observó que los aceites esenciales constituyen una fuente importante de productos naturales con efecto farmacológico en el sistema respiratorio, justificando el uso popular de las especies vegetales en el tratamiento del asma, tos y en la promoción de la expectoración.

Palabras clave: Aceites esenciales. Asma. Tos. Expectoración. Efecto espasmolítico.

\section{INTRODUÇÃO}

Os óleos essenciais ou óleos voláteis encontrados especialmente em plantas aromáticas, variam de odor e sabor, que são diferenciados de acordo com os tipos e quantidade de constituintes presentes nos óleos ${ }^{(1)}$.

Os óleos essenciais possuem composição química extremamente complexa devido à presença de constituintes altamente funcionalizados, que estão agrupados em duas classes químicas: terpenoides e fenilpropanoides. Os terpenoides são extremamente variáveis, sendo que já foram isolados aproximadamente 55.000 constituintes, possuindo diferentes esqueletos de carbono e uma grande variedade de derivados oxigenados, incluindo álcoois, ésteres, aldeídos, cetonas, éteres, peróxidos e fenóis(2).

Os terpenoides, de modo geral, classificam-se de acordo com o esqueleto de carbono. Os menores terpenos contêm uma única unidade de isopreno (C5) e são chamados de hemi-terpenos ou meio-terpenos. À medida que as unidades de isopreno vão sendo metabolizadas pelo organismo da planta, a partir dos diversos estímulos genéticos e epigenéticos, são originados os monoterpenos (C10), sesquiterpenos (C15), diterpenos (C20), triterpenos (C30), tetraterpenos (C40), dentre outras classificações de acordo com a quantidade de carbonos na molécula e/ou unidades de isopreno presentes ${ }^{(3,4)}$.

As propriedades farmacêuticas de plantas aromáticas são parcialmente atribuídas aos óleos essenciais, termo que foi usado pela primeira vez no século XVI por Paracelsus, nomeando o componente efetivo de uma droga com o nome de "quinta essencial". Em meados do século 20, o papel dos óleos essenciais foi reduzido ao uso em perfumaria, cosméticos e aromas alimentares, enquanto houve declínio na utilização em preparações farmacêuticas ${ }^{(5)}$.

Os óleos essenciais, além de serem produtos utilizados na área de aromas, cosméticos e alimentos, têm despertado o interesse farmacológico de alguns setores da indústria farmacêutica. Isso porque esse produto natural pode estar em abundância na natureza e apresenta diversos efeitos farmacológicos desde atividade repelente, anti-inflamatória, passando por atividade antiasmática, ansiolítica e atividade anticâncer ${ }^{(6,7)}$. Os óleos essenciais têm sido conhecido por possuir atividade antioxidante e antimicrobiana, servindo assim como aditivos naturais em alimentos e produtos alimentares ${ }^{(1)}$. Além disso, alguns óleos essenciais são utilizados na medicina popular para o tratamento de doenças respiratórias.

Diante disso, o objetivo deste artigo é relacionar as espécies vegetais que são fontes de óleos essenciais com efeito farmacológico no trato respiratório, seja em modelos experimentais in vitro com órgãos isolados ou in vivo que envolvam atividade antiasmática, antitussígena e/ou expectorante. 


\section{PROCEDIMENTOS METODOLÓGICOS}

Realizou-se uma busca por artigos completos publicados entre o período de janeiro de 2001 a junho de 2016, com a utilização das bases de dados especializadas Pubmed, SCOPUS e Biblioteca Virtual de Saúde. Os descritores utilizados para a busca foram, "essential oil and spasmolytic", "essential oil and airway smooth muscle", "essential oil and antiasthmatic", "essential oil and antitussive", "essential oil and cough", "essential oil and mucolytics" ou "essential oil and expectorant".

Os critérios de escolha foram artigos completos e que apresentavam as palavras-chave no título ou resumo, sendo feita essa verificação após análise do texto completo para que não houvesse falha na seleção. Aqueles que não estavam de acordo com os critérios de inclusão e as palavras-chave não foram identificadas, não possuíam acesso ao artigo completo, repetições ou artigos de revisão foram excluídos.

\section{RESULTADOS E DISCUSSÃO}

Foram identificados 868 artigos (Tabela 1) indexados nas bases de dados utilizadas, com Pubmed apresentando 256, SCOPUS com 520 e Biblioteca Virtual de Saúde com 92 artigos. Desse total, 846 manuscritos foram excluídos da avaliação por não preencherem os critérios para inclusão. Na Figura 2 é possível observar a esquematização da revisão a partir das bases de dados. Assim, foram incluídos 22 artigos ( $n=22$ ), em um período compreendido de 2001 a 2016, conforme demonstrado na Tabela 2 (APÊNDICE A).

Tabela 1. Quantidade de artigos indexados em bases de dados relatando atividade espasmolítica, antiasmática, antitussígena e/ou expectorante de óleos essenciais

\begin{tabular}{c|c|c|c|c}
\hline Base de dados & $\mathbf{N}^{\circ}$ total de trabalhos & $\mathbf{N}^{\circ}$ de trabalhos excluídos & Período consultado & \multirow{2}{*}{ Data da Pesquisa } \\
\hline PUBMED & 256 & 244 & & \\
\hline SCOPUS & 520 & 510 & \multirow{2}{*}{2001 a 2016} & \multirow{2}{*}{$23 / 09 / 16$} \\
BVS & 92 & 89 & & \\
\hline Total & $\mathbf{8 6 8}$ & $\mathbf{8 4 3}$ & & \\
\hline
\end{tabular}

Fonte: Elaborada pelos autores, 2017. 
Figura 1: Fluxograma da realização da revisão a partir das bases de dados

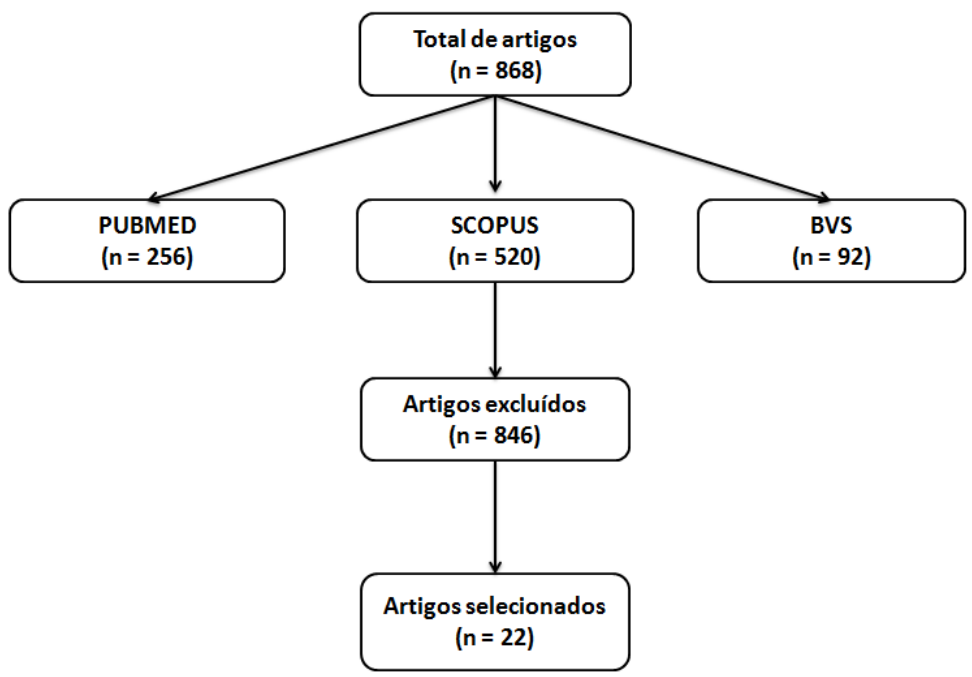

Fonte: Elaborada pelos autores, 2017.

\subsection{Modelos experimentais para avaliação de drogas sobre o sistema respiratório}

A contração da musculatura lisa das vias aéreas constitui um fator de extrema relevância na regulação da resistência ao fluxo do ar para os pulmões, dependendo principalmente da produção de segundos mensageiros importantes. O aumento da concentração citosólica de cálcio $\left(\left[\mathrm{Ca}^{2+}\right]_{i}\right)$, por exemplo, seja por liberação dos estoques intracelulares, via inositol trisfosfato $\left(\mathrm{IP}_{3}\right)$, receptores de rianodina (RyR), proteína cinase dependente de cálcio (PKC), ou pela entrada através de canais para Ca+ localizados na membrana plasmática são fatores chave para muitas respostas celulares ${ }^{(8)}$. Nesse sentido, a utilização de órgãos isolados, como traqueia e brônquios, para avaliação do potencial relaxante de novas substâncias constitui uma estratégia importante na identificação de compostos antiasmático.

O ensaio utilizando sensibilização de animais com ovoalbumina (OVA) constitui um dos mais utilizados para avaliar o quadro inflamatório, remodelagem das vias aéreas e hiper-responsividade brônquica ${ }^{(9)}$. Além de elucidar os mecanismos das respostas imunológicas e inflamatórias da asma, a resposta aguda a alérgenos tem sido amplamente utilizados para a identificação de novos alvos e a investigação de novos potenciais tratamentos para controlar a inflamação alérgica ${ }^{(10)}$. A utilização do modelo experimental com OVA permite avaliar o papel de interleucinas (IL) como a IL-4, IL-5 e IL-13 que são encontradas em respostas típicas de linfócitos T helper do tipo 2 (Th2) e são responsáveis pela progressão da doença alérgica e continuidade da inflamação, bem como hiper-responsividade das vias aéreas ${ }^{(11)}$.

Outro ensaio utilizado para avaliação de drogas com efeito no sistema respiratório corresponde à indução de pleurisia em roedores. Nesse modelo experimenta,l o zymosan, um extrato insolúvel de parede celular (proteínas, quitina, ß-glucana, mananas e lipídeos), obtido da levedura Saccharomyces cerevisae, é injetado por via intrapleural e induz inflamação na pleura dos animais, a partir da liberação do fator de necrose tumoral (TNF), IL-1, IL-6 e migração de neutrófilos, o que permite uma avaliação da atividade anti-inflamatória de compostos atuantes no sistema respiratório ${ }^{(12,13)}$. Além disso, pode-se observar uma 
migração e ativação de macrófagos que produzem mediadores pró-inflamatórios, incluindo os eicosanoides, tais como as prostaglandinas (PGs) e os leucotrienos (LTs), fator ativador de plaquetas (PAF), e citocinas inflamatórias, contribuindo ainda mais para o desenvolvimento de inflamação ${ }^{(14)}$.

A asma promove também outros sinais além da inflamação, por exemplo, tosse. Para avaliar atividade antitussígena existem modelos experimentais que utilizam capsaicina, alguns solventes orgânicos e, em especial, o ácido cítrico. A inalação de ácido cítrico estimula todo o trato respiratório, a partir da cavidade nasal até os brônquios e, por conseguinte, não só induz tosse, mas também espirros. Essa substância tem a capacidade de promover a tosse estimulando as fibras $C$ ou $A \delta$, sendo considerada uma metodologia efetiva para análise de tosse ou teste de antitussígenos ${ }^{(15)}$. Outros estudos propuseram o mecanismo tussígeno do ácido cítrico a partir da estimulação de mastócitos ou ação direta sobre as fibras $C$ com envolvimento de taquicininas e sua ação sobre as fibras $A \delta$ adaptativas que atuam sobre o sistema nervoso central ${ }^{(16)}$.

A hipersecreção mucosa é outro fator preponderante para debilitação dos indivíduos com asma, por causa da redução da luz dos brônquios e aumento da resistência à passagem do ar, principalmente por ser uma secreção espessa(17,18). Um modelo para avaliar a secreção dessas células mucosas a partir de estímulos farmacológicos é utilizando o indicador vermelho de fenol. Essa metodologia avalia a quantidade de vermelho de fenol secretado pelas células mucosas nas vias aéreas, permitindo uma avaliação sobre um aumento ou redução dessa secreção promovida por fármacos ou produtos naturais. Os mecanismos pelos quais o vermelho de fenol é secretado ainda não estão estabelecidos, no entanto, sabe-se que um aumento de secreção do indicador correlaciona a uma maior possibilidade de secreção aquosa, sendo benéfico para os indivíduos por mudar as características biofísicas do muco tornando-os mais fluidos ${ }^{(19,20,21)}$.

\subsection{Espécies vegetais contendo óleos essenciais com atividade no sistema respiratório}

Angelicae sinensis (Oliv.) Diels.: Ratos sensibilizados com OVA foram tratados com o óleo essencial de $A$. Sinensis, sendo observado os comportamentos asmáticos, funções respiratórias, níveis de IL-10 no lavado broncoalveolar (LBA) e a expressão de Foxp3 (molécula reguladora de linfócitos TCD4+) no pulmão dos animais asmáticos. O óleo apresentou melhora na função pulmonar dos ratos elevando os níveis de IL-10 no lavado broncoalveolar e a expressão de Foxp3 nos pulmões ${ }^{(22)}$.

Artemisia maritima L.: O óleo essencial de A. maritima foi utilizado em experimento in vitro utilizando traqueia isolada de coelho pré-contraída com carbacol (CCh) ou KCl. Mesmo induzindo relaxamento em ambas as situações, o óleo foi mais eficaz em relaxar o músculo liso contraído por CCh. Além disso, a partir da utilização de verapamil e isoprenalina foi sugerido o possível mecanismo de ação através de um bloqueio dos canais para cálcio e inibição da fosfodiesterase, corroborando os usos na medicina popular dessa planta para o tratamento da asma ${ }^{(23)}$.

Aster tataricus L.f.: A atividade expectorante do óleo essencial foi avaliada utilizando o modelo de secreção do marcador vermelho de fenol na traqueia de camundongo. O efeito expectorante do óleo essencial de $A$. tataricus foi associado ao componente majoritário 1-acetoxi-2-eno- $E$-4,6-decandieno ${ }^{(24)}$.

Atractylodes macrocephala Koidz:: O óleo essencial de A. macrocephala foi investigado quanto à potencial atividade expectorante em camundongos, utilizando o modelo de vermelho de fenol. Observou-se que os níveis desse indicador na traqueia dos animais tratados com o óleo essencial foram semelhantes ao controle positivo utilizado (bromexina) e ambos significativamente diferentes quando comparados ao controle negativo (solução salina)(25).

Boswellia carterii Birdwood. e Thymus vulgaris L.: O efeito antitussígeno dos óleos essenciais obtidos de $B$. carterii e T. vulgaris em camundongos foi avaliado em modelo de tosse induzida por ácido cítrico. Os animais tratados com os óleos essenciais mostraram uma diminuição significativa na frequência de tosses em comparação com o grupo que recebeu apenas 
o veículo. Quando comparado com os animais tratados com difenidramina e terbutalina, o óleo de $B$. carterii apresentou uma potência relativa de até $59 \%$ enquanto que o óleo de T. vulgaris uma potência relativa de $89 \%{ }^{(26)}$.

Boswellia sacra Flueck.: O óleo essencial de B. sacra foi avaliado em camundongos com asma induzida por OVA e observouse redução da eosinofilia, hiperplasia e responsividade brônquica, sendo significativamente diferente do grupo que recebeu apenas o veículo que tiveram esses mesmos parâmetros exacerbados. Além disso, o lavado broncoalveolar mostrou uma maior produção da citocinas Th1 (IFN- $\gamma$ ) e redução nos níveis de citocinas Th2 (IL-4, IL-5, IL-13), os níveis de IgE, dentre outros, mostrando um efeito imunomodulador desse óleo essencial(27).

Em outro estudo com $B$. sacra observou-se que camundongos também sensibilizados com OVA tiveram melhora nos parâmetros inflamatórios no tecido pulmonar após tratamento com o óleo essencial, com redução do percentual de células inflamatórias no LBA e nas células mononucleares do sangue periférico. Esses dados sugerem que o óleo essencial dessa espécie é eficaz no tratamento da asma por influenciar a resposta imunológica em diferentes tecidos ${ }^{(28)}$.

Carum copticum H.Karst.: Quatro frações do óleo essencial de C. copticum foram utilizados para avaliar o efeito relaxante em traqueia isolada de cobaias pré-contraída com metacolina ou $\mathrm{KCl}$, com a utilização do propranolol e clorfeniramina para sugerir o mecanismo de ação. A fração 1 apresentou efeito relaxante dependente da concentração, mas os resultados não apresentaram diferença significativa quando comparados aos do etanol (grupo controle). Foi observado um efeito relaxante nos diferentes grupos experimentais com as frações 2 e 3 apresentando maior potência. A fração 4 não promoveu relaxamento em quase todas as concentrações testadas nos anéis pré-contraídos com metacolina. No estudo não foi possível indicar o mecanismo de ação, apenas inferir que o carvacrol pode ser o responsável pelo efeito relaxante da fração 2 do óleo essencial(29). Conyza bonariensis (L.) Cronquist: Investigou-se o efeito do óleo essencial de C. bonariensis no modelo de inflamação pleural (pleurisia) induzida por zimosan e lipopolissacarideo (LPS). O óleo induziu redução dos leucócitos totais, células mononucleares e neutrófilos apenas nos animais que a pleurisia foi induzida por LPS(30).

Croton nepetaefolius Baill.: O óleo essencial de C. nepetaefolius foi investigado com potencial relaxante da musculara lisa de traqueia isolada de cobaias sensibilizadas ou não com OVA. Em preparações pré-contraídas com $\mathrm{KCl}$, o óleo essencial promoveu relaxamento máximo, sendo similar à aminofilina (inibidor da fosfodiesterase), sem causar alterações no potencial transmembrana. Além disso, o óleo também relaxou o músculo liso de traqueia isolada de cobaia contraído com CCh ou histamina de forma equipotente ${ }^{(31)}$.

Croton sonderianus Müll.Arg.: O efeito de duas amostras de óleo essencial de $C$. sonderianus coletados em períodos distintos de um mesmo dia foram avaliados em traqueia isolada de rato pré-contraída ou não com acetilcolina ( $\mathrm{ACh}$ ) ou $\mathrm{KCl}$. Surpreendentemente, o óleo coletado às $13 \mathrm{~h}$ induziu relaxamento, enquanto que o coletado às $21 \mathrm{~h}$ induziu contração em preparações envolvendo o tônus basal. No entanto, em contrações induzidas por $\mathrm{ACh}$ ou $\mathrm{KCl}$ ambos os óleos promoveram relaxamento do músculo liso traqueal com potências relativas diferentes. A diferença entre os efeitos observados podem ser explicado pela diferente composição química dos óleos essenciais(32).

Eucalyptus tereticornis Sm.: Em estudo realizado com músculo liso traqueal de ratos, o óleo essential de E. tereticornis potencializou a contração induzida por acetilcolina ACh ao passo que inibiu, de maneira dependente da concentração, a estimulação contrátil eletromecânica do $\mathrm{KCl}$. Os componentes majoritários $\alpha$ e $\beta$-pineno também potencializaram as contrações induzidas tanto por ACh quanto por $\mathrm{KCl}^{(33)}$.

Gerbera piloselloides L.: O óleo essencial das raízes foi avaliado em camundongos nos modelos experimentais de atividade antitussígena e expectorante. Na dose de $2.000 \mathrm{mg} / \mathrm{kg}$, administrado por via intragástrica, foi observada modulação da tosse e expectoração a partir dos tratamentos com o óleo essencial.

Lavandula angustifolia Bubani: Camundongos sensibilizados com OVA foram tratados com o óleo essencial de L. angustifolia, sendo observado redução do nível de células inflamatórias no BAL, principalmente eosinófilos, bem como reduziu a hiper- 
responsividade induzida por metacolina. Além disso, foi observado que o óleo inibiu a hiperplasia das células musculares lisas das vias aéreas dos camundongos e reduziu a expressão de IL-4, IL-5 e Muc5B ${ }^{(35)}$.

Lippia dulcis Trevir: O estudo com óleo essencial de L. dulcis foi realizado com brônquios secundários de cobaias, que foram cumulativamente contraídos com carbacol ou histamina na ausência ou presença do óleo. Observou-se que na presença do óleo houve redução do efeito máximo induzido por ambos os agonistas, sugerindo que o efeito pode estar relacionado a um antagonismo não-competitivo(36)

Lippia thymoides Mart \& Schauer: Quatro amostras dos óleos essenciais obtidos sazonalmente de L. thymoides apresentaram baixa eficácia em relaxar segmentos de traqueia isolada de cobaia pré-contraídas com CCh, com todas as amostras dos óleos apresentando efeito máximo de cerca de $50 \%{ }^{(37)}$.

Mentha piperita Linn: O óleo essencial de M. piperita induziu relaxamento do músculo liso de traqueia isolada de ratos pré-contraído com CCh. Ao avaliar o possível mecanismo de ação, observou-se que o efeito relaxante do óleo essencial foi reduzido na presença de indometacina, L-NAME, tetrametilamônio e hexametônio, sugerindo a participação de postanoides, óxido nítrico, canais para $\mathrm{K}^{+}$e liberação de ACh no efeito do óleo essencial de M. piperita ${ }^{(38)}$.

Mentha pulegium L.: $O$ efeito relaxante do óleo essencial de M. pulegium foi avaliado em músculo liso traqueal de ratos pré-contraídos com ACh ou $\mathrm{KCl}$. Como o óleo apresentou maior eficácia em reduzir as contrações induzidas por acoplamento eletromecânico, foi investigada a participação do fluxo de $\mathrm{Ca}^{2+}$ proveniente do meio extracelular e sugerindo que o possível mecanismo de ação desse óleo envolve o bloqueio da entrada de $\mathrm{Ca}^{2+}$ do meio extracelular e que, em parte, pode estar relacionado ao componente majoritário pulegona ${ }^{(39)}$.

Nepeta cataria L.: O óleo essencial de N. cataria induziu relaxamento de músculo liso traqueal isolado de cobaias pré-contraídos com CCh ou KCl. Ao investigar o possível mecanismo de ação, observou-se que a presença do óleo essencial potencializou o relaxamento provocado pela isoprenalina, efeito similar ao apresentado quando a isoprenalina foi adicionada na presença de papaverina, indicando que a atividade do óleo essencial de $N$. cataria está relacionado inibição da fosfodiesterase e bloqueio do fluxo de $\mathrm{Ca}^{2+(40)}$.

Ocimum micranthum Willd.: Ratos sensibilizados com OVA foram utilizados para avaliar o efeito do óleo essencial de O. micranthum em músculo liso de traqueia isolada. Observou-se que as contrações do músculo liso induzidas pela adição cumulativa de $\mathrm{CCh}$ ou $\mathrm{KCl}$ foram reduzidas na presença do óleo, indicando que houve redução da hiper-responsividade dos animais sensibilizados ${ }^{(41)}$.

Phellolophium madagascariensis Baker.: O óleo essencial de P. madagascariensis foi utilizado em estudos in vitro de traqueia isolada de cobaias. Foi observado que o óleo é capaz de promover o relaxamento do músculo liso das vias aéreas pré-contraído com histamina, e esse relaxamento pode ser inibido na presença de propranolol, indicando que a atividade relaxante apresentada pelo óleo essencial provavelmente é mediada por receptores $\beta$-adrenérgicos ${ }^{(42)}$.

Piper betel Linn.: Em anéis de traqueia isolada de cobaia, a presença do óleo essencial de $P$. betel inibiu significativamente as contrações do músculo liso induzidas por histamina, quando comparado com as preparações que não continham o óleo. Além disso, a administração oral do óleo foi capaz de proteger as cobaias ao desafio com histamina que ocasiona convulsão por broncoconstrição(43).

Pistacia integerrima J. L. Stewart: O óleo essencial de P. integerrima foi testado como potencial inibidor da lipoxigenase (LOX) e parâmetros envolvendo inflamação pulmonar induzida por LPS em ratos, mostrando-se efetivo ao reduzir a atividade de LOX. O total de células inflamatórias no LBA, neutrófilos, atividade da mieloperoxidase (MPO), o nível de nitrito/nitrato (avaliação indireta da produção de óxido nítrico), proteínas totais e níveis de albumina foram reduzidos significativamente nos animais tratados com o óleo essencial. Nos ratos que foram tratados com o óleo na dose de $30 \mathrm{mg} / \mathrm{kg}$, a análise histopatológica dos pulmões revelou que não apresentaram anormalidade tecidual, similar ao observado nos animais não sensibilizados com LPS. 
Além disso, o óleo essencial conseguiu abolir as convulsões broncoprovocativas em cobaias sensibilidadas e desafiadas por inalação de OVA ${ }^{(44)}$.

Pterodon polygalaeflorus Benth.: O óleo essencial de $P$. polygalaeflorus foi avaliado em traqueia isolada de rato em tônus basal ou pré-contraída com ACh, $\mathrm{KCl}$ ou serotonina (5-HT), com a utilização de ferramentas farmacológicas para avaliação do seu possível mecanismo de ação. Observou-se que o óleo inibiu apenas as contrações induzidas por 5- $\mathrm{HT}$ e $\mathrm{KCl}$, sendo que em preparações pré-incubadas com Ba²+ e na presença de tetraetilamônio, o óleo inibiu as contrações induzidas por ACh, sugerindo que o mecanismo de ação relaxante pode estar relacionado com ativação de canais para $\mathrm{Ca}^{2+(45)}$.

Rosmarinus officinalis L.: A avaliação da atividade anti-inflamatória do óleo essencial de R. officinalis em modelo de pleurisia induzida por carragenina em ratos, indicou que os animais tratados com o óleo na dose de $500 \mathrm{mg} / \mathrm{kg}$ por via oral tiveram uma significativa redução do exudato inflamatório e de leucócitos na cavidade pleural(46).

Xylopia langsdorfiana St. Hilaire \& Tulasne: O óleo essencial de $X$. langsdorfiana apresentou eficácia em relaxar os anéis de traqueia isolada de cobaia, observado efeito máximo quando foi utilizado na concentração de $243 \mu \mathrm{g} / \mathrm{mL}^{(47)}$.

Zingiber officinale Roscoe: O óleo essencial de $Z$. officinale teve a atividade espasmolítica avaliada em traqueia isolada de ratos pré-contraída com CCh, seguido da triagem do possível mecanismo de ação. Observou-se que o óleo foi capaz de relaxar a musculatura lisa das vias aéreas, sendo mais potente e eficaz do que um dos seus componentes majoritários, o citral. Além disso, foi mostrado que os efeitos do óleo essencial têm como possível mecanismo de ação, agonismo $\beta_{2}$-adrenérgico, pois na presença de propranolol houve redução significativa do efeito relaxante tanto do óleo essencial quanto do citral(48).

\section{CONCLUSÃO}

A análise dos artigos indicou que há poucos estudos envolvendo a avaliação de óleos essenciais sobre o efeito relaxante na musculatura lisa das vias aéreas e atividade antiasmática em modelos animais. Além disso, espécies vegetais de distintas famílias botânicas foram investigadas, indicando que não há concentração dos estudos farmacológicos dos óleos essenciais em determinados gêneros. Considerando a grande diversidade química dos óleos essenciais e a possibilidade de atuação desses produtos naturais em diversas vias de sinalização no sistema respiratório, as plantas aromáticas constituem uma importante fonte de substâncias de interesse farmacológico visando ao tratamento de doenças respiratórias, além de justificar o uso dessas plantas na medicina popular.

\section{REFERÊNCIAS}

1. Tongnuanchan P, Benjakul S. Essential oils: extraction, bioactivities, and their uses for food preservation. J. Food. Sci. 2014;79(7):1231-1249.

2. Rehman R, Hanif MA, Mushtag Z, Al-Sadi AM. Biosynthesis of essential oils in aromatic plants: a review. Food .Rev. Int. 2016;32(2):117-160.

3. Biswas KK, Foster AJ, Aung T, Mahmoud SS. Essential oil production: relationship with abundance of glandular trichomes in aerial surface of plants. Acta Physiol. Plant. 2009;31(1):13-19.

4. Maffei ME. Sites of synthesis, biochemistry and functional role of plant volatiles. S. Afr. J. Bot. 2010;76(4):612-631.

5. Edris AE. Pharmaceutical and therapeutic potentials of essential oils and their individual volatile constituents: a review. Phytother. Res. 2007;21(4):308-323. 
6. Ajikumar PK, Tyo K, Carlsen S, Mucha O, Phon TH, Stephanopoulos G. Terpenoids: opportunities for biosynthesis of natural product drugs using engineered microorganisms. Mol. Pharm. 2008;5(2):167-190.

7. Gautam N, Mantha AK, Mittal S. Essential oils and their constituents as anticancer agents: a mechanistic view. Biomed. Res. Int. 2014;2014:1-23.

8. Albuquerque AA, Carvalho MT, Evora PM, de Nadai TR, Celotto AC, Evora PR. In vitro reactivity ("organ chamber") of guinea pig tracheal rings-methodology considerations. Ann. Transl. Med. 2016;4(11):216.

9. Kumar RK, Herbert C, Foster PS. The "classical" ovalbumin challenge model of asthma in mice. Curr. Drug. Targets. 2008;9(6):485-494.

10. Nials AT, Uddin S. Mouse models of allergic asthma: acute and chronic allergen challenge. Dis. Model. Mech. 2008;1(45):213-220.

11. Zosky GR, Sly PD. Animal models of asthma. Clin Exp Allergy. 2007;37(7):973-988.

12. Utsunomiya I, Ito M, Oh-ishi S. Generation of inflammatory cytokines in zymosan-induced pleurisy in rats: TNF induces IL-6 and cytokine-induced neutrophil chemoattractant (CINC) in vivo. Cytokine. 1998;10(12):956-963.

13. Magnani M, Castro-Gómez RJH. ß-glucana de Saccharomyces cerevisiae: constituição, bioatividade e obtenção. Semin: Ciênc Agrár. 2008;29(3):631-650.

14. Yuhki K, Ushikubi F, Naraba H, Ueno A, Kato H, Kojima F et al. Prostaglandin $I 2$ plays a key role in zymosan-induced mouse pleurisy. J. Pharmacol. Exp. Ther. 2008;325(2):601-609.

15. Tanaka M, Maruyama K. Mechanisms of capsaicin- and citric-acid-induced cough reflexes in guinea pigs. J. Pharmacol. Sci. 2005;99(1):77-82

16. Lai $\mathrm{YL}, \mathrm{Wu} \mathrm{LL}$, Lin TY, Lin CH. The role of mast cells in citric acid-induced airway constriction and cough. Chin. J. Physiol. 2009;52(5):332-338.

17. Dhar R. Role of mucolytics in wet cough. Supplement to J Assoc Physicians India. 2013;61(5 Suppl):23-27.

18. Rogers DF. Mucoactive agents for airway mucus hypersecretory diseases. Respir .Care. 2007;52(9):1176-1197.

19. Engler H, Szelenyl I. Tracheal phenol red secretion, a new method for screening mucosecretolytic compounds. J. Pharmacol. Methods. 1984;11(3):151-157.

20. Liu W, Cheng X, Wang Y, Li S, Zheng T, Gao Y et al. In vivo evaluation of the antitussive, expectorant and bronchodilating effects of extract and fractions from aerial parts of Peganum harmala linn. J. Ethnopharmacol. 2015;162:79-86.

21. Song KJ, Shin YJ, Lee KR, Lee EJ, Suh YS, Kim KS. Expectorant and antitussive effect of Hedera helix and Rhizoma coptidis extracts mixture. Yonsei Med. J. 2015;56(3):819-824.

22. Wang ZW, Li RK, Lin XY, Ren Y, Liu XF, Cheng XL et al. [Effect of Volatile Oil of Radix Angelicae sinensis on experimental asthma in rats]. Zhongguo Ying Yong Sheng Li Xue Za Zhi. 2015;31(2):107-110.

23. Shah AJ, Gilani AH, Abbas K, Rasheed M, Ahmed A, Ahmad VU. Studies on the chemical composition and possible mechanisms underlying the antispasmodic and bronchodilatory activities of the essential oil of Artemisia maritima L. Arch Pharm Res. 2011;34(8):1227-1238.

24. Yang B, Xiao YQ, Liang RX, Wang RJ, Li W, Zhang C et al. [Studies on expectorant compounds in volatile oil from root and rhizome of Aster tataricus]. Zhongguo Zhong Yao Za Zhi. 2008;33(3):281-3. Chinese. 
25. Wang X, Linghui L, Xiaoku R, Deqiang D, Bin L, Bingyou $Y$ et al. What caused the changes in the usage of Atractylodis Macrocephalae Rhizoma from ancient to current times? J Nat Med. 2016;70(1):36-44.

26. Taha KF, Hetta MH, Bakeer WI, Yassin NAZ, Ibrahim BMM, Hassan MES. Comparative phytochemical and pharmacological study of antitussive and antimicrobial effects of boswellia and thyme essential oils. Der Pharma Chemica. 2016;8(1):67-83.

27. Lee HY, Yun MY, Kang SM. Anti-inflammatory effect of Boswellia sacra (Franckincense) essential oil in a mouse model of allergic asthma. Korean J Microbiol Biotechnol. 2008;36(4):343-352.

28. Lee HY, Kim KR, Kang SM. The effect of nebulized frankincense essential oil in an ova-induced allergic asthma mouse model. Korean J Microbiol Biotechnol. 2010;38:93-104.

29. Boskabady MH, Ramazani M, Tabei T. Relaxant effects of different fractions of essential oil from Carum copticum on guinea pig tracheal chains. Phytother Res. 2003;17(10):1145-1149.

30. Souza MC, Siani AC, Ramos MF, Lima OJM, Henriques MG. Evaluation of anti-inflammatory activity of essential oils from two Asteraceae species. Pharmazie. 2003;58(8):582-586.

31. Magalhães PJ, Lahlou S, Santos MAV, Pradines TL, Leal-Cardoso JH. Myorelaxant effects of the essential oil of Croton nepetaefolius on the contractile activity of the guinea-pig tracheal smooth muscle. Planta Med. 2003;69(9):874-877.

32. Pinho-da-Silva L, Mendes-Maia PV, Garcia TMN, Cruz JS, Morais SM, Coelho-de-Souza AN et al. Croton sonderianus essential oil samples distinctly affect rat airway smooth muscle. Phytomedicine. 2010;17(10): 721-725.

33. Lima FJ, Brito TS, Freire WB, Costa RC, Linhares MI, Sousa FC et al. The essential oil of Eucalyptus tereticornis, and its constituents $a$ - and $\beta$-pinene, potentiate acetylcholine-induced contractions in isolated rat trachea. Fitoterapia. 2010;81(6):649-655.

34. Tang XJ, Huang HR, Fang TZ, Yang CP, Zhang Y, Wu BH et al. [The antitussive and de-sputum essential substance from the root of Gerbera piloselloides and its analytical compositions]. Zhongguo Zhong Yao Za Zhi. 2003;28(5):426-429. Chinese.

35. Ueno-lio T, Shibakura M, Yokota K, Aoe M, Hyoda T, Shinohata R et al. Lavender essential oil inhalation suppresses allergic airway inflammation and mucous cell hyperplasia in a murine model of asthma. Life Sci. 2014;108(2):109-115.

36. Görnemann T, Nayal R, Pertz HH, Melzig MF. Antispasmodic activity of essential oil from Lippia dulcis Trev. J Ethnopharmacol. 2008;117(1):166-169.

37. Silva FS, Menezes PM, Sá PG, Oliveira AL, Souza EA, Almeida JR et al. Chemical composition and pharmacological properties of the essential oils obtained seasonally from Lippia thymoides. Pharm. Biol. 2016;54(1):25-34.

38. Sousa AA, Soares PM, Almeida AN, Maia AR, Souza EP, Assreuy AM. Antispasmodic effect of Mentha piperita essential oil on tracheal smooth muscle of rats. J Ethnopharmacol. 2010;130(2):433-436.

39. Soares PM, de Freitas Pires A, de Souza EP, Assreuy AM, Criddle DN. Relaxant effects of the essential oil of Mentha pulegium L. in rat isolated trachea and urinary bladder. J. Pharm Pharmacol. 2012;64(12):1777-1784.

40. Gilani AH, Shah AJ, Zubair A, Khalid S, Kiani J, Ahmed A, et al. Chemical composition and mechanisms underlying the spasmolytic and bronchodilatory properties of the essential oil of Nepeta cataria L. J Ethnopharmacol. 2009;121(3):405411.

41. Pinho JP, Silva AS, Pinheiro BG, Sombra I, Bayma JC, Lahlou S et al. Antinociceptive and antispasmodic effects of the essential oil of Ocimum micranthum: potential anti-inflammatory properties. Planta Med. 2012;78(7):681-685. 
42. Andriamanantoanina H, Ramaroson L, Raminosoa T, Ratsimbason M, Casabianca H, Grenier-Loustalot MF. Chemical composition and biological activity of essential oil of Phellolophium madagascariensis Baker (Umbellifereae). J. Essent. Oil. Res. 2006;18(2):231-233.

43. Hajare R, Darvhekar VM, Shewale A, Patil V. Evaluation of antihistaminic activity of Piper betel leaf in guinea pig. Afr. J. Pharm Pharmacol. 2011;5(2):113-117.

44. Shirole RL, Shirole NL, Kshatriya AA, Kulkarni R, Saraf MN. Investigation into the mechanism of action of essential oil of Pistacia integerrima for its antiasthmatic activity. J. Ethnopharmacol. 2014;153(3):541-551.

45. Evangelista GL, Souza ANC, Santos CF, Leal-Cardoso JH, Lopes EAB, Santos MV et al. Essential oil of Pterodon polygalaeflorus inhibits electromechanical coupling on rat isolated trachea. J. Ethnopharmacol. 2007;109(3):515-522.

46. Takaki I, Bersani-Amado LE, Vendruscolo A, Sartoretto SM, Diniz SP, Bersani-Amado CA et al. Anti-inflammatory and antinociceptive effects of Rosmarinus officinalis L. essential oil in experimental animal models. J. Med. Food. 2008;11(4):741746.

47. Correia AC, Ferreira TF, Martins IR, Macêdo CL, Monteiro FS, Costa VC et al. Essential oil from the leaves of Xylopia langsdorfiana (Annonaceae) as a possible spasmolytic agent. Nat. Prod. Res. 2015;29(10):980-984.

48. Mangprayool T, Kupittayanant S, Chudapongse N. Participation of citral in the bronchodilatory effect of ginger oil and possible mechanism of action. Fitoterapia. 2013;89:68-73.

Submetido em: 07/12/2016

Aceito em: 05/07/2017

Publicado em: 30/09/2017 


\begin{tabular}{|c|c|c|c|c|c|c|c|c|}
\hline & 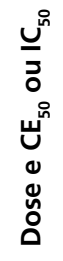 & 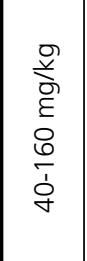 & 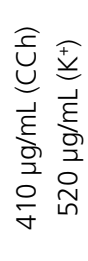 & ' & 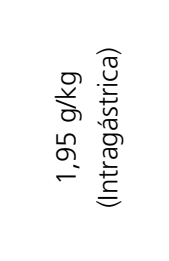 & 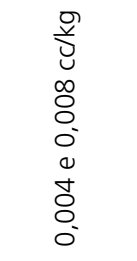 & 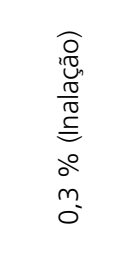 & 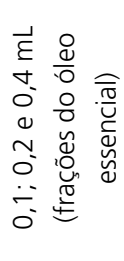 \\
\hline & 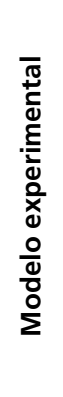 & 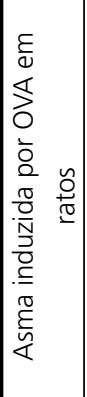 & 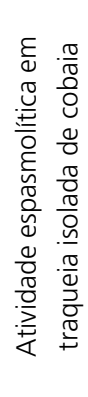 & 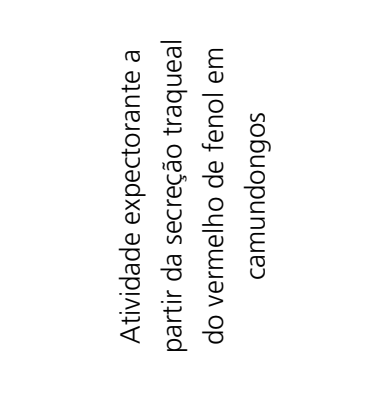 & 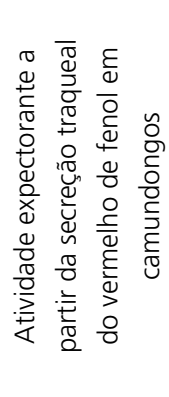 & 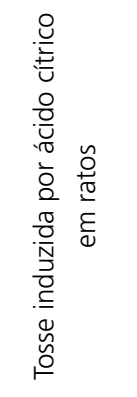 & 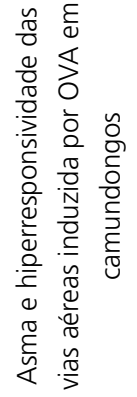 & 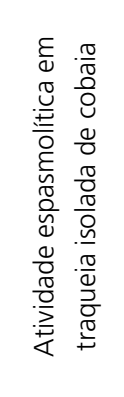 \\
\hline 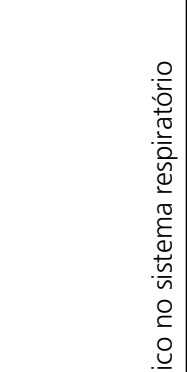 & 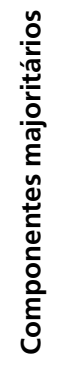 & ' & 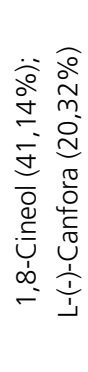 & 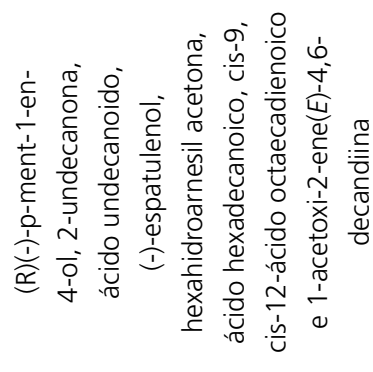 & 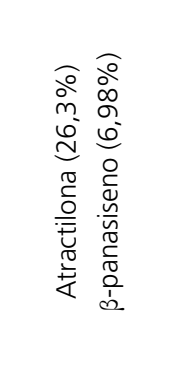 & 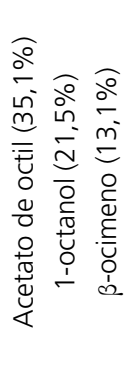 & ' & 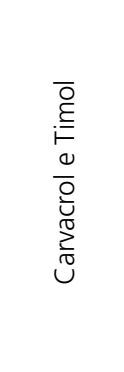 \\
\hline 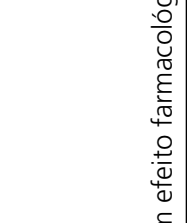 & 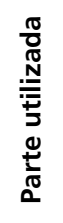 & 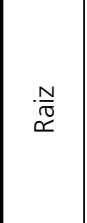 & ' & 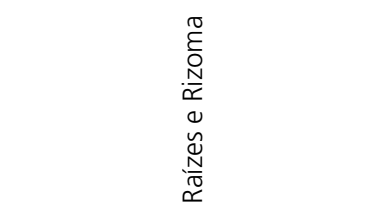 & $\begin{array}{l}\stackrel{\tilde{E}}{\mathcal{D}} \\
\stackrel{\mathcal{N}}{\sim}\end{array}$ & & ' & 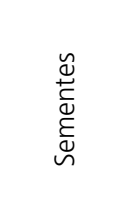 \\
\hline 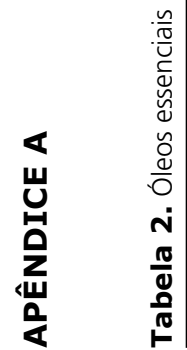 & $\begin{array}{l}\stackrel{\frac{\pi}{2}}{\frac{\pi}{2}} \\
\frac{\pi}{2}\end{array}$ & 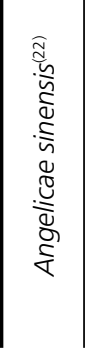 & 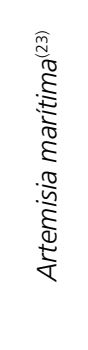 & 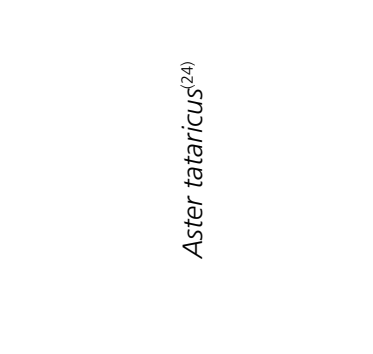 & 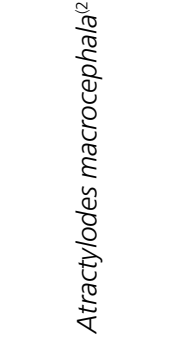 & 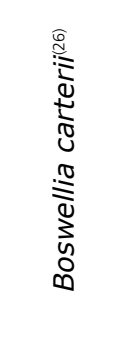 & 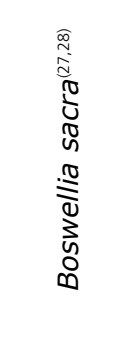 & 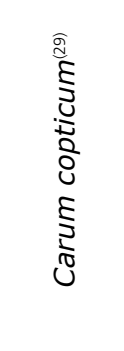 \\
\hline
\end{tabular}




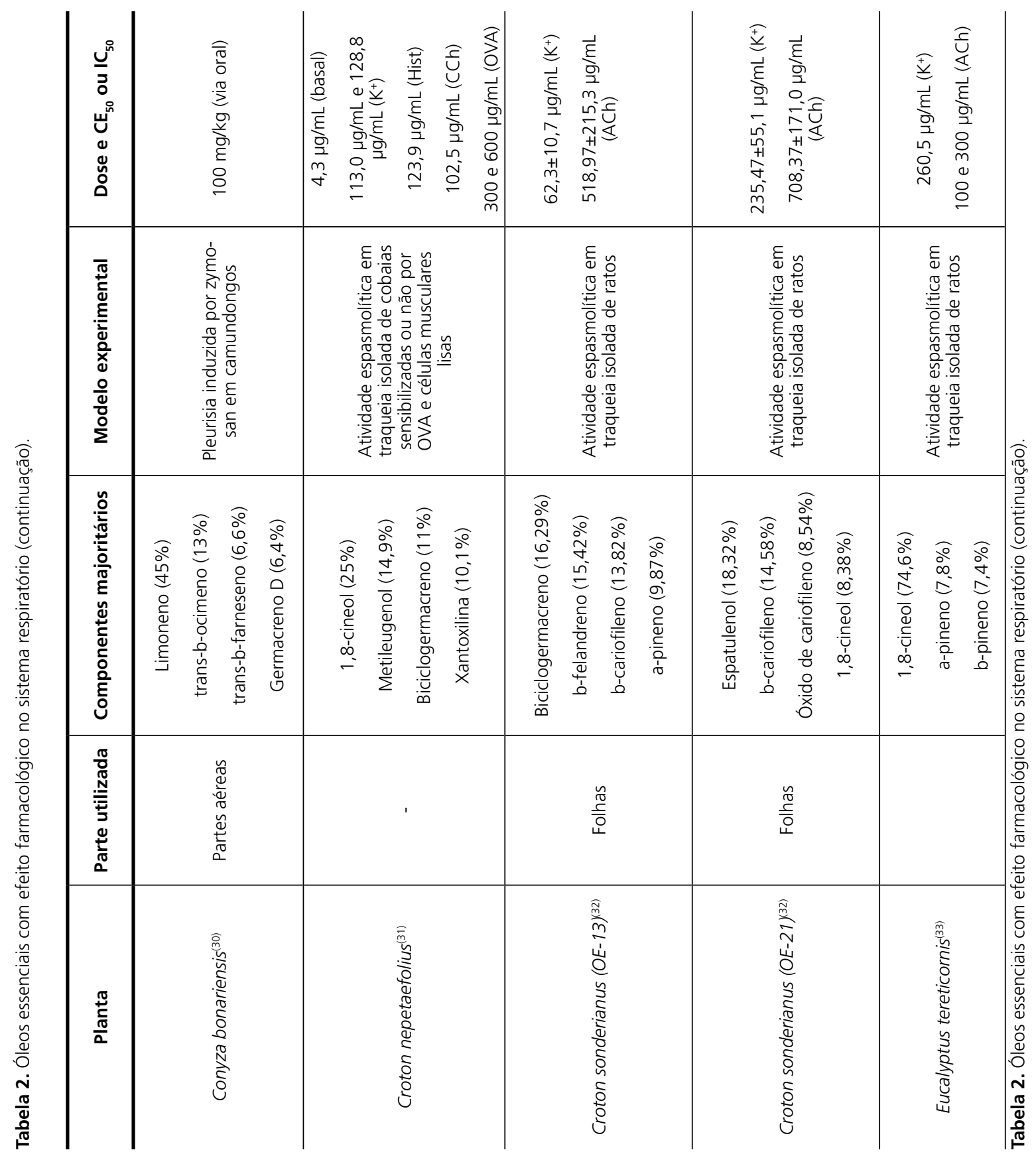




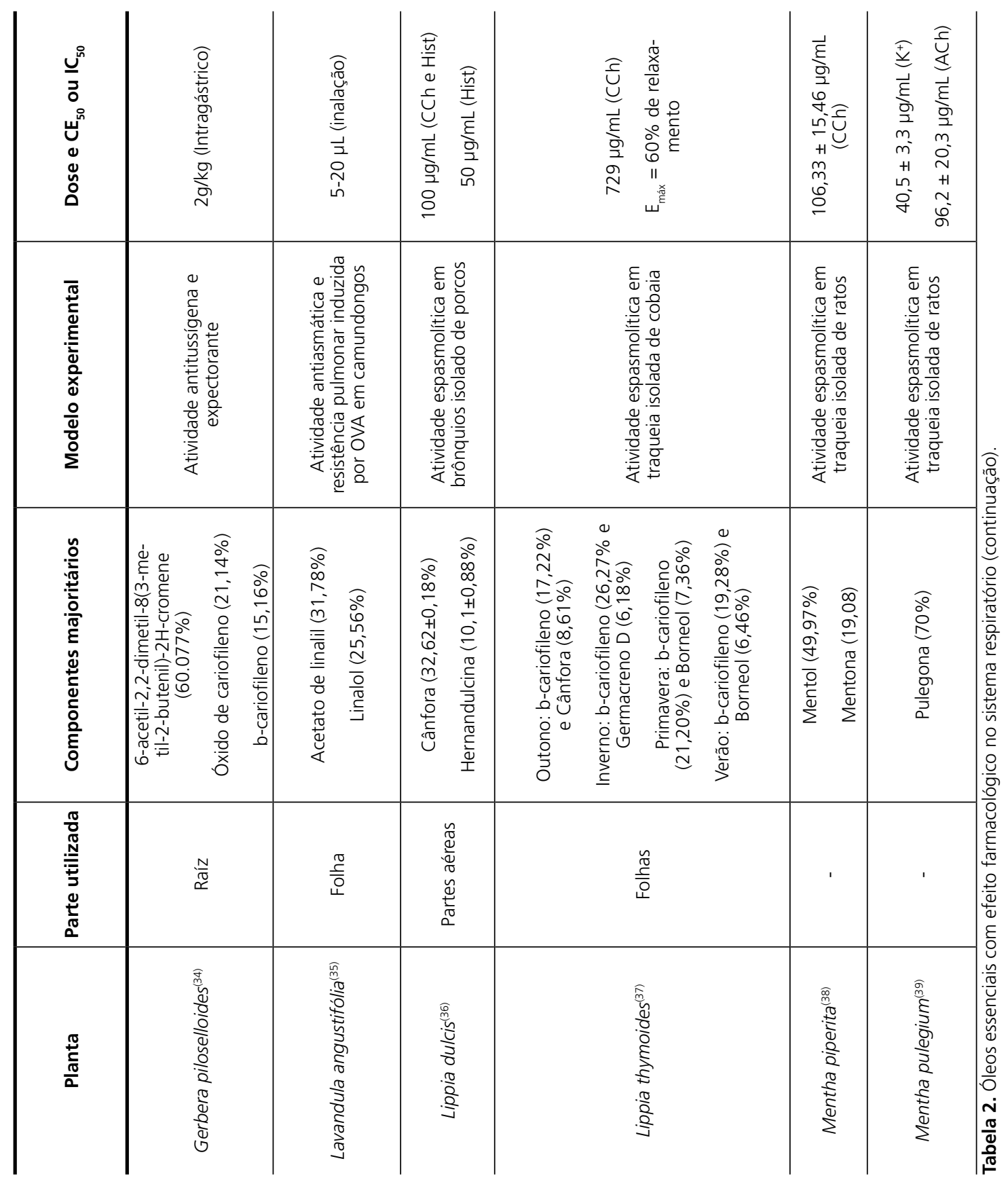




\begin{tabular}{|c|c|c|c|c|c|}
\hline 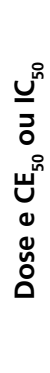 & 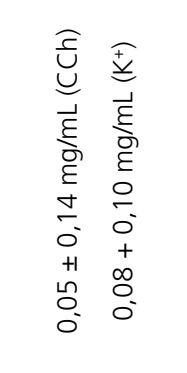 & 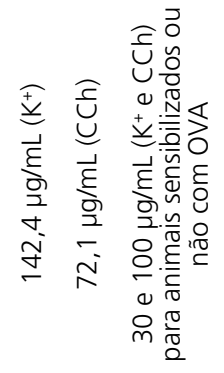 & $\begin{array}{l}\frac{1}{2} \\
\stackrel{8}{\circ}\end{array}$ & 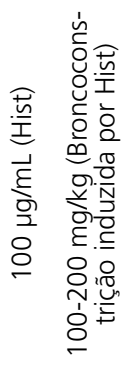 & 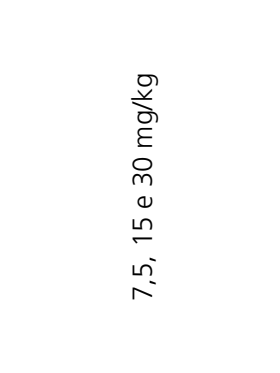 \\
\hline 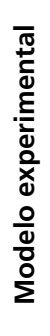 & 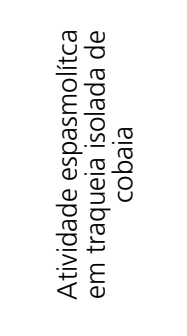 & 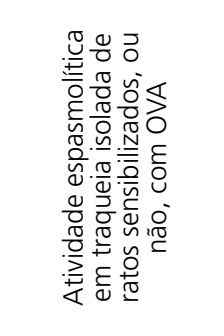 & 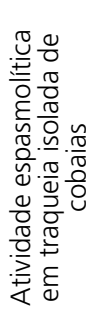 & 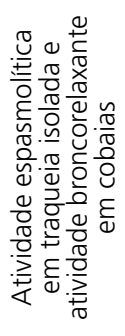 & 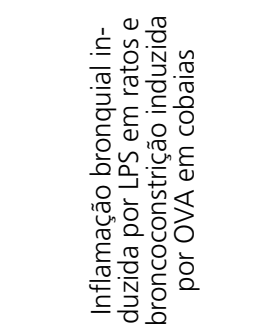 \\
\hline 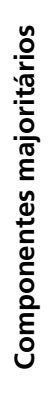 & 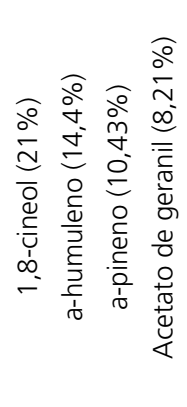 & 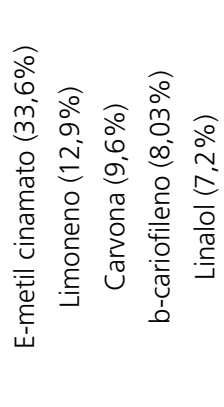 & 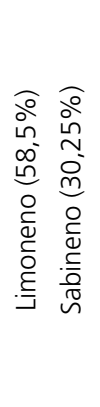 & ' & 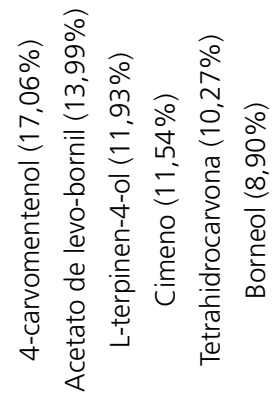 \\
\hline 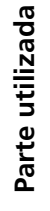 & & ' & 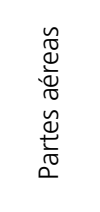 & $\frac{\stackrel{0}{\overline{0}}}{\frac{0}{4}}$ & $\begin{array}{l}\stackrel{\tilde{0}}{\frac{\pi}{\pi}} \\
\stackrel{\widetilde{J}}{0}\end{array}$ \\
\hline $\begin{array}{l}\stackrel{\frac{\pi}{2}}{\frac{\pi}{a}} \\
\frac{\pi}{a}\end{array}$ & 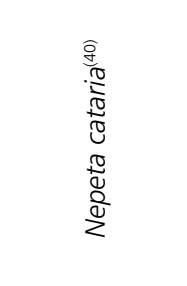 & 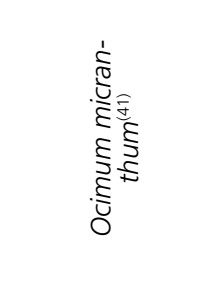 & 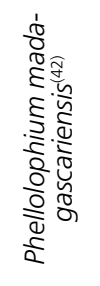 & 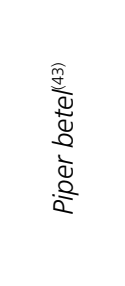 & 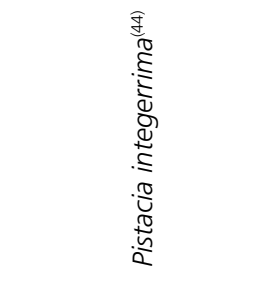 \\
\hline
\end{tabular}




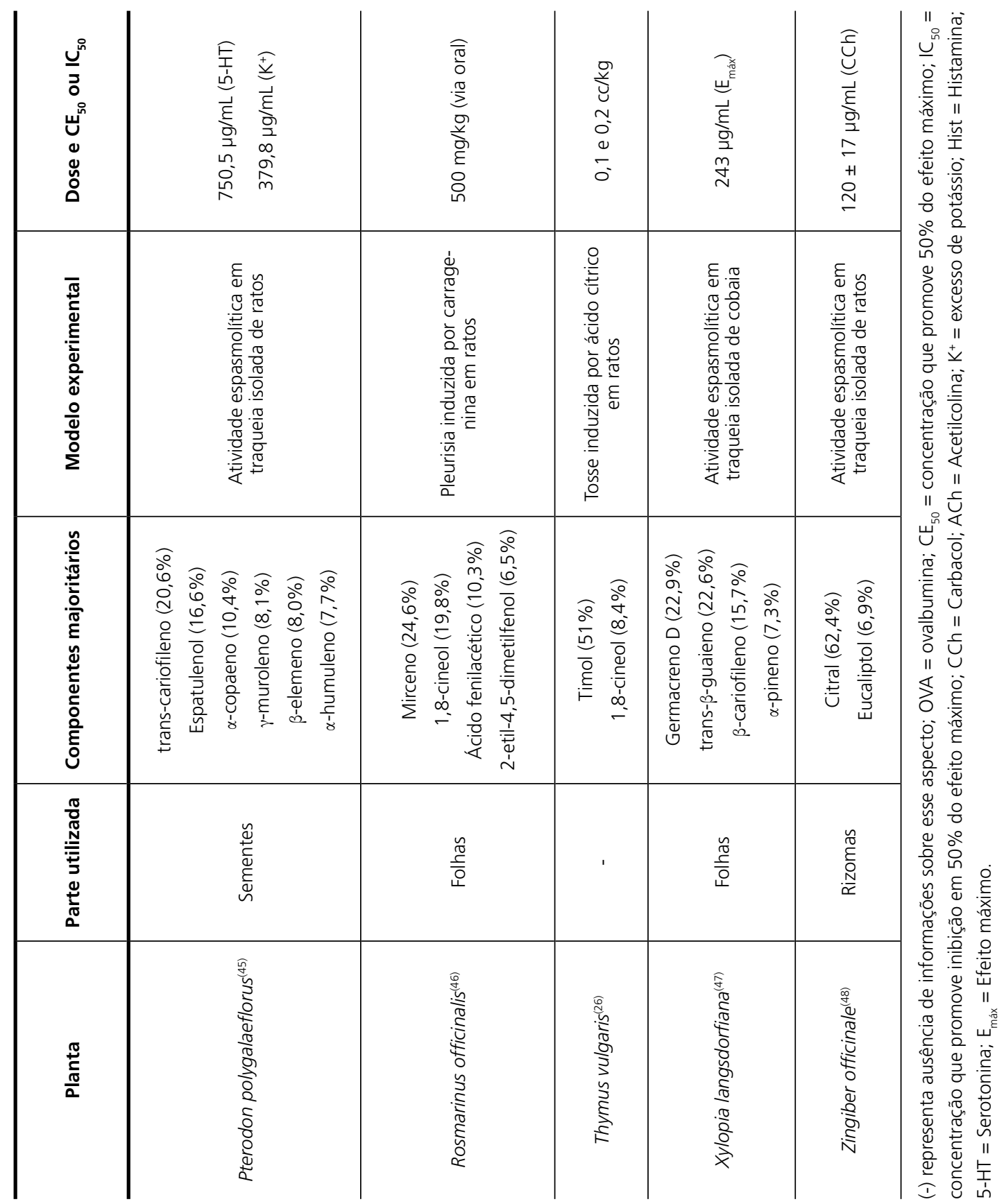

